\title{
Devitrification of Bi- and Ga-containing germanium-based chalcogenide glasses
}

\author{
L. Saturday ${ }^{1}$, C. Johnson ${ }^{1}$, A. Thai ${ }^{1}$, J. Szlęzak ${ }^{2}$, Ya. Shpotyuk ${ }^{2}$, R. Golovchak ${ }^{1 *}$ \\ ${ }^{I}$ Department of Physics and Astronomy, Austin Peay State University, Clarksville, TN 37044, USA \\ ${ }^{2}$ Centre for Innovation and Transfer of Natural Sciences and Engineering Knowledge, \\ University of Rzeszow, Rzeszow, 35-959, POLAND
}

\begin{abstract}
Novel $\mathrm{Bi}_{\mathrm{x}} \mathrm{Ga}_{\mathrm{y}}\left(\mathrm{GeSe}_{4}\right)_{50-(\mathrm{x}+\mathrm{y}) / 2}\left(\mathrm{GeTe}_{4}\right)_{50-(\mathrm{x}+\mathrm{y}) / 2}$ glasses are proposed as perspective materials, having potential to combine thermo-electric effect, rare earth solubility, different types of conductivity and topological insulating behaviour in one medium. Thermal stability and nonisothermal crystallization kinetics of these materials are studied by differential scanning calorimetry, X-ray diffraction and Raman scattering techniques. Crystallization of at least five different phases is assumed due to the fit of experimental DSC curves. The Dietzel criterion of glass stability is determined and the activation energy for each crystallization process is calculated. The applicability of Johnson-Mehl-Avrami (JMA) equation to describe the crystallization kinetics is verified in these glasses.
\end{abstract}

Keywords: chalcogenide glasses; crystallization; kinetics; XRD; Raman spectroscopy

\footnotetext{
* Corresponding author: Department of Physics and Astronomy, Austin Peay State University, Clarksville, TN-37044, USA.

E-mail address: holovchakr@apsu.edu (R. Golovchak). Tel.: 1-931-2216361
} 


\section{Introduction}

Chalcogenide glasses $(\mathrm{ChG})$ are considered as the most convenient and inexpensive media for applications in modern photonics, combining high IR transparency, excellent fiber drawing capability and largest optical nonlinearities reported to date [1,2]. Many tellurium- and seleniumbased compositions are being engineered in order to improve and optimize the performance of photonic devices [2-4]. Conventionally, this goal is achieved through the variation in composition of complex $\mathrm{ChG}$, which allows tailoring their physical properties to match the requirements. As a result of such approach, number of complex Te- and Se-based compositions are proposed for application in far-IR optics, waveguiding for space telecommunication, optical and bio-sensing technologies, phase-change memory devices [1-6]. However, significant enhancement of $\mathrm{ChG}$ initial properties is also possible due to design on the nanoscale. The nanoscale modification of $\mathrm{ChG}$ is especially intriguing, since nano-engineering opens the possibility to design hybrid materials combining two or more useful properties (mechanical, electronic, optical, magnetic) in one functional medium [7]. In this regard, several chemical elements like $\mathrm{Bi}$ and $\mathrm{Ga}$ are found to modify $\mathrm{ChG}$ at the nanoscale by triggering local crystallization of amorphous matrix at the elevated temperatures or under the external influences (light, pressure, radiation, etc.) [8-10]. Therefore, crystallization kinetics of multicomponent Biand Ga-containing $\mathrm{ChG}$ is of significant practical importance.

In this work we report thermal stability and crystallization kinetics studies for the complex glasses of Bi-Ga-(GeSe 4$)-\left(\mathrm{GeTe}_{4}\right)$ family, which are anticipated to be used as a host matrix having potential to combine thermo-electric effect, rare earth solubility, different types of conductivity and topological insulating behaviour in one medium. X-ray diffraction (XRD) and Raman spectroscopy are used to identify the products of devitrification at each phase transition. 


\section{Materials and Methods}

The $\mathrm{Bi}_{\mathrm{x}} \mathrm{Ga}_{\mathrm{y}}\left(\mathrm{GeSe}_{4}\right)_{50-(\mathrm{x}+\mathrm{y}) / 2}\left(\mathrm{GeTe}_{4}\right)_{50-(\mathrm{x}+\mathrm{y}) / 2}(\mathrm{y}=5, \mathrm{x}=1,5,10)$ glasses were prepared by conventional melt-quench route from high-purity ( $5 \mathrm{~N}$ or more) chemical precursors. Appropriate amounts of $\mathrm{Ga}, \mathrm{Bi}, \mathrm{Ge}$, Se and $\mathrm{Te}$ (with total weight close to $25 \mathrm{~g}$ ) were vacuum sealed in silica tube of $10 \mathrm{~mm}$ diameter. Then, the ampoules were heated up to $900{ }^{\circ} \mathrm{C}$ with $2{ }^{\circ} \mathrm{C} / \mathrm{min}$ rate in a rocking furnace, homogenized for $12 \mathrm{~h}$ and quenched into water from $700{ }^{\circ} \mathrm{C}$. To relieve the mechanical strains appeared during rapid quenching, the samples were additionally annealed for $3 \mathrm{~h}$, at $\sim 160{ }^{\circ} \mathrm{C}$. All the obtained materials were in glassy form as established by the absence of crystalline reflexes in XRD patterns, except the $\mathrm{Bi}_{10} \mathrm{Ga}_{5} \mathrm{Ge}_{17} \mathrm{Se}_{34} \mathrm{Te}_{34}$ sample, which showed the presence of crystalline inclusions already after quenching (Fig. 1). The actual composition of the prepared materials, as checked by energy-dispersive X-ray analysis, was found to be $\mathrm{Bi}_{1.1} \mathrm{Ga}_{5.2} \mathrm{Ge}_{18.6} \mathrm{Se}_{37.4} \mathrm{Te}_{37.7}(\mathrm{R} 1), \mathrm{Bi}_{5.5} \mathrm{Ga}_{5.5} \mathrm{Ge}_{18.9} \mathrm{Se}_{36.8} \mathrm{Te}_{33.3}$ (R2) and $\mathrm{Bi}_{10} \mathrm{Ga}_{5.8} \mathrm{Ge}_{18.4} \mathrm{Se}_{36.9} \mathrm{Te}_{28.9}$ (R3), which was very close to the desired glasses $\left(\mathrm{Bi}_{1} \mathrm{Ga}_{5} \mathrm{Ge}_{18.8} \mathrm{Se}_{37.6} \mathrm{Te}_{37.6}, \mathrm{Bi}_{5} \mathrm{Ga}_{5} \mathrm{Ge}_{18} \mathrm{Se}_{36} \mathrm{Te}_{36}\right.$ and $\mathrm{Bi}_{10} \mathrm{Ga}_{5} \mathrm{Ge}_{17} \mathrm{Se}_{34} \mathrm{Te}_{34}$, respectively).

XRD patterns were collected on powdered samples at room temperature in the angular range $10^{\circ}<2 \theta<100^{\circ}$, using Bruker D8 Advance (with $\mathrm{Cu}$ anode $\lambda=1.5406 \AA$ as $\mathrm{X}$-Ray source) X-ray diffractometer in Bragg-Brentano geometry.

Each sample was thermally analyzed using differential scanning calorimeter model TA Instruments 2920 (calibrated with Ga, In, Sn and Pb as standard elements) at heating rates of 2, $5,10,15$, and $20 \mathrm{~K} / \mathrm{min}$. Bulk chunks of approximately $20 \mathrm{mg}$ of each glass were measured in aluminum crucibles under nitrogen atmosphere. The differential scanning calorimetry (DSC) measurements were repeated minimum 3 times for each heating rate, each time using different 
sample of the same composition to assure repeatability of the results. The measurement protocol included 2 runs at each heating rate for each sample. The first run revealed the glass-tosupercooled liquid transition and crystallization peaks, while the second run of fully crystallized sample provided us with a confident baseline, which was then subtracted from the first run curve. The repeatable DSC data sets were analyzed with Origin software to fit the crystallization peaks with Gaussian and Fraser-Suzuki distributions. Although the latter is considered to be more appropriate in the case of thermal analysis data [11], we report both for comparison. Each of these fitted curves was then integrated and normalized using Origin's intrinsic functions to get a fraction of crystallized volume $(\alpha)$.

Raman spectroscopy data were measured in $50-550 \mathrm{~cm}^{-1}$ range with Horiba Xplora confocal microscope, using $785 \mathrm{~nm}$ laser for excitation. Raman spectra of amorphous R1 and R2 samples were difficult to obtain initially, because of the crystallization tendency under the influence of probe laser beam. Therefore, appropriately reduced laser intensity and long acquisition time have been used to minimize any beam-induced crystallization. The acquisition time was in the range of $100-200 \mathrm{~s}$ and the power of excitation beam was $\sim 0.07 \mathrm{~mW} \cdot \mu \mathrm{m}^{-2}-$ low enough to avoid photo-induced crystallization proper to these materials under the above laser wavelength. Spectra collected for each sample from four different regions within the freshly cut edge of surface area were averaged to increase the confidence in the data.

\section{Results and discussion}

Thermograms obtained with DSC for the investigated $\mathrm{Bi}_{1} \mathrm{Ga}_{5} \mathrm{Ge}_{18.8} \mathrm{Se}_{37.6} \mathrm{Te}_{37.6}(\mathrm{R} 1)$, $\mathrm{Bi}_{5} \mathrm{Ga}_{5} \mathrm{Ge}_{18} \mathrm{Se}_{36} \mathrm{Te}_{36}(\mathrm{R} 2)$ and $\mathrm{Bi}_{10} \mathrm{Ga}_{5} \mathrm{Ge}_{17} \mathrm{Se}_{34} \mathrm{Te}_{34}(\mathrm{R} 3)$ samples after background and baseline subtraction are shown in Fig. 2. The onset values of glass transition temperature $T_{g}$, peak value 
of the first crystallization temperature $T_{c}$ (determined for $10 \mathrm{~K} / \mathrm{min}$ runs), as well as Dietzel criterion of glass stability [12] are given in Table 1. It is obvious, that glass becomes less stable with Bi addition and finally Bi-based crystallites appear in $\mathrm{R} 3$ sample with 10 at.\% of Bi (Fig. 1). Nevertheless, $T_{g}$ of all the investigated samples is higher than $160{ }^{\circ} \mathrm{C}$, which makes them attractive compositions for applications in many IR instruments.

The DSC curves of R1 and R2 glasses exhibit two families of exothermic peaks, which correspond to the crystallization regions (Fig. 2$)$, namely "low-temperature" $\left(\sim 210-300{ }^{\circ} \mathrm{C}\right)$ and "high-temperature" ( 320-370 $\left.{ }^{\circ} \mathrm{C}\right)$. Gaussian or Fraser-Suzuki fits of DSC heating curves (Fig. 3) both suggest the crystallization of at least five different phases in R2 sample (consistent also with the first derivative analysis of DSC curves): three phases in the first and two in the second region. It should be noted however, that shape of real crystallization peaks is usually more complicated than Gaussian form due to asymmetry and depends on the crystallizing phase $[11,13]$. On the other side, Fraser-Suzuki function can fit asymmetric peaks better [11], but there is a risk of missing weak shoulder peaks in the case of complex overlapped signals. Thus, contrary to Gaussian fit, which needs 5 peaks in order to achieve an acceptable goodness of fit for DSC signal of R1 sample, the use of Fraser-Suzuki function can reduce a number of peaks to 3. However, we believe there should be the same number of peaks as in the sample R2, since R1 is amorphous and belongs to the same chemical family. So, because of lower concentration of $\mathrm{Bi}$ in $\mathrm{R} 1$, the related crystallization peaks can be masked by the crystallization of Ga-based compounds. Therefore, we report the results from both Gaussian and Fraser-Suzuki fitting procedures, using 5 peaks to fit the DSC curves of R1 and R2 samples. In any case, the proposed fittings should be considered as idealized models, which, however, are our best estimates (due to the overlapped crystallization processes of unknown nature) to determine the crystallization 
temperatures and activation energies of crystallization processes. From comparison of DSC curves for R1 and R2 samples we can argue that peak I is dominated by the crystallization of Bibased compounds (like $\mathrm{Bi}_{2} \mathrm{Te}_{3}, \mathrm{Bi}_{2} \mathrm{Se}_{3}$ or $\mathrm{Bi}_{2} \mathrm{SeTe}_{2}$ ). Such asignment is consistent with the reports of other authors investigating crystallization in Ge-Se-Te-Bi system [14,15], where crystallization of $\mathrm{Bi}_{2} \mathrm{Se}_{3}$ phase was found in $260-310{ }^{\circ} \mathrm{C}$ range depending on the heating rate $[15$, 16]. The second peak II observed in the first region of DSC curves does not change much with Bi addition. Therefore, it can be attributed to the crystallization of Ga-based phases (like $\mathrm{Ga}_{2} \mathrm{Te}_{3}$, $\mathrm{Ga}_{2} \mathrm{Se}_{3}$ or mixed), which where identified in crystallized products of Ge-Te-Ga [17], Ga-As-Se [18] and Ga-As-Se-Te [18,19] glasses as well. The broad peak labeled III in the first region of DSC curves, the most probably, includes crystallization of trigonal Te and various metastable $\mathrm{Ga} / \mathrm{Ge}-\mathrm{Te}$ phases, like $\mathrm{GeTe}_{2}$ identified in binary Ge-Te system $[17,20]$. The second family of crystallization processes occurred in high-temperature region (peaks IV-VI) can be related to the crystallization of stable $\mathrm{Ge}-\mathrm{Te}\left(\mathrm{GeTe}, \mathrm{Ge}_{2} \mathrm{Te}_{3}\right.$ or $\left.\mathrm{Ge}_{17} \mathrm{Te}_{83}\right)$ [17, 21], Ge-Se (GeSe, $\mathrm{GeSe}_{2}$, $\mathrm{Ge}_{4} \mathrm{Se}_{9}$ ) or mixed phases. Despite the crystallization of $\mathrm{GeSe}_{2}$ phase is reported for Ge-Se-Bi [22] and $\mathrm{Ge}_{20} \mathrm{Se}_{50} \mathrm{Te}_{30}$ [23] glasses in a similar temperature range, the crystallization of this phase in pure Ge-Se system usually is observed at higher temperatures $\left(\sim 530{ }^{\circ} \mathrm{C}\right)[24,25]$. Although there is a possibility that existence of Ga-/Bi-based crystallites and addition of Te can facilitate the crystallization of $\mathrm{GeSe}_{2}$ phase, triggering it at lower temperatures, the obtained Raman and XRD data have little support of its formation in present materials after heat treatment. The main $A_{1}$ breathing mode at $\sim 210 \mathrm{~cm}^{-1}$ of corner-shared $\mathrm{GeSe}_{4 / 2}$ tetrahedra in crystalline $\mathrm{GeSe}_{2}$ [24,26-28] is not observed in any of the heat-treated samples (Fig. 4). Instead, a feature at $\sim 190 \mathrm{~cm}^{-1}$ is the closest one which can be associated with $\mathrm{GeSe}_{2}$ crystallites formation, but it is weak and observed only in R2 and R3 samples after heat treatments (Fig. 4). There is, of course, a 
possibility that addition of $\mathrm{Bi}$ leads to a significant decrease in the intensity of Raman signal from $\mathrm{GeSe}_{2}$ vibrations as reported in [27], but a reason for such effect in the investigated materials is not clear. Moreover, small difference between the Raman spectra recorded for R1(R2) samples after crystallization at $300(250){ }^{\circ} \mathrm{C}$ and $400{ }^{\circ} \mathrm{C}$ suggests superior crystallization of the telluride-based phases.

The interpretation of the rest features in Raman spectra is not straightforward because of the variety of possible structural units which vibrational modes strongly overlap. The obtained Raman spectra (Fig. 4) are dominated by the vibrations from Ga-, Bi- and Ge-based telluride complexes, which for vitreous R1 and R2 samples are observed as broad bands in the ranges of $\sim 80-110 \mathrm{~cm}^{-1}, \sim 120-160 \mathrm{~cm}^{-1}$ and $\sim 180-210 \mathrm{~cm}^{-1}$ [29]. The band at $\sim 80-90 \mathrm{~cm}^{-1}$ is usually observed in a single-crystal $\alpha-G e T e$ and can be attributed to $\Gamma_{3}(\mathrm{E})$ mode of rhombohedrally deformed rocksalt structure [30] or bending modes of $\mathrm{GeTe}_{4}$ tetrahedra [31]. The band peaking at $\sim 125-140 \mathrm{~cm}^{-1}$ is characteristic to $\mathrm{A}_{1}$ mode of corner-shared $\mathrm{GeTe}_{4}$ tetrahedra and lighter $\mathrm{Ge}_{2} \mathrm{Te}_{3}$ complexes [31]. The vibrational modes of edge-shared $\mathrm{GeTe}_{4}$ tetrahedra usually give rise to a band in Raman spectra at $\sim 160 \mathrm{~cm}^{-1}$ [32]. Nevertheless, the position of all these peaks can be shifted in the investigated materials because of Te substitution in $\mathrm{GeTe}_{4}$ tetraherdra with $\mathrm{Se}, \mathrm{Bi}$ or $\mathrm{Ga}$ atoms, forming mixed $\mathrm{GeTe}_{4-\mathrm{n}}(\mathrm{Se} / \mathrm{Bi} / \mathrm{Ga})_{n}$ units [33]. The third family of low-intensity peaks within $\sim 180-210 \mathrm{~cm}^{-1}$ range, most probably, originate from $\mathrm{GeSe}_{4}$ tetrahedra, where one or two Se atoms are replaced with Te. The Raman peaks of Ga-based complexes, expected at $\sim 105$, $\sim 118, \sim 155, \sim 250, \sim 290 \mathrm{~cm}^{-1}$ (for $\mathrm{Ga}_{2} \mathrm{Se}_{3}[34,35]$ ) and $\sim 117, \sim 130 \mathrm{~cm}^{-1}$ (breathing modes of $\mathrm{GaTe}_{4 / 2}$ units [36]), are hardly observed in Raman spectra of the investigated glasses because of the low Ga concentration (5 at.\%) and their strong overlap with other modes of telluride-based units. The crystalline $\mathrm{Bi}_{2} \mathrm{Se}_{3}$ usually gives rise to Raman bands at $\sim 75, \sim 130$ and $\sim 175 \mathrm{~cm}^{-1}$ [37- 
39], while $\mathrm{Bi}_{2} \mathrm{Te}_{3}$ Raman active modes lie at $\sim 62, \sim 104, \sim 134 \mathrm{~cm}^{-1}$ [40,41]. All of them can be assumed in the experimental Raman spectra of the heat treated R1-R3 samples (Fig. 4), but they cannot be unambiguously resolved without appropriate theoretical modelling due to a strong overlap with other modes of Ga- and Ge-based structural units.

Further refinement of the crystallizing phases can be achieved from the analysis of XRD patterns of the samples before and after heat treatments (Fig. 5). According to PDF database [42], the phases crystallizing in the first "low-temperature" region (peaks I-III) can be identified as $\mathrm{Te}, \mathrm{Bi}_{2} \mathrm{Se}_{\mathrm{n}} \mathrm{Te}_{3-\mathrm{n}}(\mathrm{n}=0,0.5,1,1.5,2)$ and $\mathrm{Ga}_{2} \mathrm{Se}_{2} \mathrm{Te}$ compounds. The intensities of the corresponded reflexes in the XRD patterns from Bi-based complexes correlate well with $\mathrm{Bi}$ content in the samples (Fig. 5). The second "high-temperature" region (peaks IV-VI) can be associated with the crystallization of $\mathrm{GeSeTe}, \mathrm{GaGeTe}$ and $\mathrm{GeBi}_{2} \mathrm{Te}_{4}$ phases, identified using PDF database [42]. The recognized phases are in agreement with the obtained Raman spectra, dominated by a signal from pure telluride (but not pure germanium tellurides) and mixed telluride/selenide complexes, and showing lack of pure germanium selenide crystallites in the structure. On the basis of the performed analysis, it can be speculated that the most rapidly crystallizing phase (peak V in DSC curves) corresponds to GeSeTe crystallites.

The activation energies $\left(E_{a}\right)$ calculated using Ozawa's plot $[43,44](\ln q v s$ 1000/T) are given in Figs 6, 7 and 8 for R1, R2 and R3 samples, respectively. Increase in Bi concentration as from R1 to R2 sample leads to general increase of activation energies of I-III peaks (Figs. 6, 7), no matter which function (Gaussian or Fraser-Suzuki) was used for fitting (the only more or less significant difference, caused by the choice of fitting function, is obtained for $E_{a}$ values of peak III). This can be explained by the fact that crystallization of R2 glass starts at lower temperatures than that of R1 glass (Fig. 2), where the viscosity of supercooled liquid is generally higher. The 
higher viscosity means more constraints for structural rearrangements needed for crystallization to occur. Therefore, if crystallization of R1 glass starts at higher temperatures (Fig. 2), the lower viscosity of supercooled liquid allows easier structural rearrangements and, consequently, smaller activation energies. On the other hand, the activation energies for peaks IV-V of R3 sample is almost twice smaller than for peaks IV-V of R1 and R2 samples. The easier crystallization in "high-temperature" region of R3 sample in comparison to R1 or R2 can be explained by a pre-existence of crystallized Bi- and Ga-based phases, which facilitate seeds fromation for the germanium telluride/selenide crystallites.

The crystallization kinetics as studied with DSC are usually analyzed with JohnsonMehl-Avrami (JMA) nucleation-growth model [45,46]. However, the JMA equation for nonisothermal conditions is valid only if a certain number of criteria are satisfied: the entire nucleation process takes place during the early stages of the transformation, and becomes negligible afterward; the overall crystallization rate is defined only by the temperature and does not depend on the previous thermal history $[47,48]$. Fundamental kinetic equations for nonisothermal crystal growth from preexisting nuclei have been developed by Ozawa [43,44] and a simple method of kinetic analysis of DSC data for these processes has been proposed [45,46]:

$$
\frac{d \alpha}{d t}=A f(\alpha) e^{\left(-\frac{E_{a}}{R T}\right)}
$$

where $\alpha$ is fraction of crystallized volume

$$
\alpha=\frac{1}{\Delta H_{c}} \int_{0}^{T} \phi d T
$$


Here $\phi$ is the specific heat flow measured with DSC (W/g) and $\Delta H_{c}$ is the total enthalpy change associated with the crystallization process; the pre-exponential factor $A$ and activation energy $E_{a}$ are kinetic parameters that should not depend on the temperature $T$ and $\alpha$; and

$$
f(\alpha)=m(1-\alpha)[-\ln (1-\alpha)]^{1-1 / m}
$$

is an algebraic expression of the JMA model $[45,46]$.

It has been demonstrated that the JMA exponent $m$ is a characteristic parameter linked to crystal forming morphology. In particular, $m \sim 1$ means predominant surface crystallization, while $m \sim 3$ corresponds to three-dimensional bulk crystallization [49]. A simple test for the applicability of JMA model according to the above-mentioned criteria is proposed by Malek [45]. It is based on the analysis of probe functions:

$$
\begin{aligned}
& y(\alpha)=\phi e^{\left(-\frac{E_{a}}{R T}\right)} \\
& z(\alpha)=\phi T^{2}
\end{aligned}
$$

In the case of non-isothermal crystallization, for JMA equation to be valid the maximum of the $z(\alpha)$ function should occur around $\alpha=0.63 \pm 0.02$ value.

Although the obtained Gaussian fitting curves can be used to estimate the activation energies with more or less acceptable accuracy (see Figs. 6-8), they cannot be used for probe $z(\alpha)$ functions at all, because Gaussian curves are symmetric and would give $z(\alpha)$ maximum always at $\alpha \sim 0.5$. Therefore, fitting with Fraser-Suzuki function should be used for these purposes. Alternatively, it can be seen from Figs. 2,3 that peak V dominates each DSC signal in the "high-temperature" crystallization range of all samples. So, it is also possible to use a real 
shape of DSC signal $\phi$ to calculate $z(\alpha)$ for peak V. Both approaches have been used to plot $z(\alpha)$ functions for peaks $\mathrm{V}$ in order to check the applicability of JMA model at least in the second crystallization region. As seen from Fig. 9, the maxima for most $z(\alpha)$ functions occur within the range of $0.6<\alpha<0.7$, which includes the $\alpha=0.63$ value. Taking into account a reasonable accuracy achievable with DSC instrument when rapid crystallization processes such as the one associated with peak $\mathrm{V}$ are measured (causes scattering in the data points due to thermal inertia of the instrument), it is likely that JMA equation is applicable to the description of crystallization kinetics for at least peak $\mathrm{V}$ of bulk $\mathrm{Bi}_{\mathrm{x}} \mathrm{Ga}_{\mathrm{y}}\left(\mathrm{GeSe}_{4}\right)_{50-(\mathrm{x}+\mathrm{y}) / 2}\left(\mathrm{GeTe}_{4}\right)_{50-(\mathrm{x}+\mathrm{y}) / 2}$ materials. If so, it is possible to estimate the JMA exponent $m$ using the double-logarithm plots (Fig. 10) [45]. The obtained $m$ values for the peaks V of R1-R3 samples are, in general, higher than 4, which is character for the crystallization processes occurring with increasing nucleation rate [50].

One of the important parameters for practical applications of the investigated $\mathrm{ChG}$ as, for example, medium for the phase-change memory is the crystallization rapidity. In order to quantify it, an "index of crystallization rapidity" $(I C R)$, which defines crystallization rapidity as a determined ratio between the peak height and width, can be calculated [51]:

$$
I C R=\ln (M H / F W H M)
$$

where $M H$ is the maximum height of the peak (in $\mathrm{W} \cdot \mathrm{g}^{-1}$ ) and $F W H M$ is the full width at the half maximum of the peak (in $\left.{ }^{\circ} \mathrm{C}\right)$.

The logarithmical scale is used in order to properly accommodate the large changes of this criterion with applied heating rate $q$. The calculated according to (6) ICR criterion for the most rapid crystallization occurred in the investigated materials (peak V) is given in Table 1 for 10 $\mathrm{K} / \mathrm{min}$ heating rate. The obtained ICR is higher than for Se-Te, Ge-Sb-Se or Bi-containing Ge- 
Sb-Te amorphous layers [51], which makes the investigated glasses $\mathrm{R}_{1}$ and $\mathrm{R}_{2}$ promising materials for phase-change memory applications, even if the disadvantage from having several crystallizing phases is taken into account.

\section{Conclusions}

At least five different crystallization processes can be assumed in $\mathrm{Bi}_{\mathrm{x}} \mathrm{Ga}_{\mathrm{y}}\left(\mathrm{GeSe}_{4}\right)_{50}$ $(\mathrm{x}+\mathrm{y}) / 2\left(\mathrm{GeTe}_{4}\right)_{50-(\mathrm{x}+\mathrm{y}) / 2}(\mathrm{y}=5, \mathrm{x}=1,5,10)$ glasses due to Gaussian and Fraser-Suzuki fits, three of which occur in $\sim 210-300{ }^{\circ} \mathrm{C}$ and the other two in $\sim 320-370{ }^{\circ} \mathrm{C}$ temperature ranges. Activation energies for crystallization in low-temperature domain increase with $\mathrm{Bi}$ concentration and with decreasing the crystallization temperatures. Raman spectra of the crystallized compounds are dominated by the vibrations of tellurium-based structural units within $80-160 \mathrm{~cm}^{-1}$ frequency domain. The XRD analysis shows crystallization of $\mathrm{Te}, \mathrm{Bi}_{2} \mathrm{Se}_{\mathrm{n}} \mathrm{Te}_{3-\mathrm{n}}(\mathrm{n}=0,0.5,1,1.5,2)$ and $\mathrm{Ga}_{2} \mathrm{Se}_{2} \mathrm{Te}$ phases in a low-temperature domain, while predominant crystallization of GeSeTe, GaGeTe and $\mathrm{GeBi}_{2} \mathrm{Te}_{4}$ phases has been assumed at higher $\left(>300{ }^{\circ} \mathrm{C}\right)$ temperatures. The JMA equation is likely to be applicable for the description of crystallization process responsible for the

peak V. A simple JMA validity check for the crystallization processes associated with other peaks is more ambiguous because of their strong overlap.

\section{Acknowledgments}

The authors acknowledge support for this research from NSF Grant DMR-1409160. 


\section{References}

[1] B. J. Eggleton, B. Luther-Davies, K. Richardson, Chalcogenide photonics, Nature Photonics 5 (2011) 141-148.

[2] Chalcogenide Glasses: Preparation, properties and application, J-L. Adam and X. Zhang (Eds), Woodhead Publishing series in Electronic and Optical Materials No.44, 2014.

[3] X. Zhang, B. Bureau, C. Boussard-Pledel, J. Lucas, P. Lucas, Glasses for seeing beyond visible, Chem. Eur. J. 14 (2008) 432-442.

[4] P. Lucas, Zh. Yang, M.K. Fah, T. Luo, Sh. Jiang, C. Boussard-Pledel, M.-L. Anne, B. Bureau, Telluride glasses for far infrared photonic applications, Optical Materials Express 3, 8 (2013) 1049.

[5] S. Cui, R. Chahal, Ya. Shpotyuk, C. Boussard, J. Lucas, F. Charpentier, H. Tariel, O. Loreal, V. Nazabal, O. Sire, V. Monbet, Z. Yang, P. Lucas, B. Bureau, Selenide and telluride glasses for midinfrared bio-sensing, Proc. SPIE 8938 (2014) 893805-1-9.

[6] D. Lencer, M. Salinga, M. Wuttig, Design rules for phase-change materials in data storage applications, Advanced Materials 23 (2011) 2030-2058.

[7] Nanorods, Nanotubes, and Nanomaterials Research Progress, W.V. Prescott and A.I. Schwartz (Eds), Nova Science Publishers Inc., 2008.

[8] Y. Liu, R. Golovchak, W. Heffner, O. Shpotyuk, G. Chen, H. Jain, Influence of Bi on topological self-organization in arsenic and germanium selenide networks, J. Materials Chemistry C 1 (2013) 6677-6683.

[9] R. Golovchak, Ya. Shpotyuk, V. Nazabal, C. Boussard-Pledel, B. Bureau, J. Cebulski, H. Jain, Study of Ga incorporation in glassy arsenic selenides by high-resolution XPS and EXAFS, J. Chem. Phys. 142 (2015) 184501-1-10. 
[10] T.-Y. Lee, Ch. Kim, Y. Kang, D.-S. Suh, Kijoon H. P. Kim, Y. Khang, Rapid crystallization of GeTe-Bi $\mathrm{Te}_{3}$ mixed layer, Appl. Phys. Lett. 92 (2008) 101908.

[11] A. Perejon, P.E. Sanchez-Jimenez, J.M. Criado, L.A. Perez-Maqueda, Kinetic Analysis of Complex Solid-State Reactions. A New Deconvolution Procedure, J. Phys. Chem. B 115 (2011) 1780-1791.

[12] A. Dietzel, Glass Structure and Glass Properties, Glasstech. Ber. 22 (1968) 41-50.

[13] J. Šesták, Science of Heat and Tthermophysical Studies: a generalized approach to thermal analysis, Elsevier, Amsterdam, Netherlands, 2005. ISBN 444519548.

[14] Zh. Wang, Ch. Tu, Y. Li, Q. Chen, The effects of Sn and Bi additions on properties and structure in Ge-Se-Te chalcogenide glass, J. Non-Cryst. Solids 191 (1995) 132-137.

[15] N. Asha Bhat, K.S. Sangunni, A consistent approach towards Bi doping mechanism in chalcogenide glasses from $C_{p}$ measurement in Ge-Se-Te-Bi system, Solid State Communications 116 (2000) 297-302.

[16] M.A. Abdel-Rahim, A.Y. Abdel-Latif, A.El-Korashy, G.A. Mohamed, Crystallization study of $\mathrm{Bi}_{5} \mathrm{Ge}_{20} \mathrm{Se}_{75}$ glass, Journal of Materials Science 30 (1995) 5737-5742.

[17] P. Petkov, V. Ilcheva, T. Petkova, P. Ilchev, Thermal Studies of GeTeGa Glasses, AIP Conf. Proc. 1203 (2010) 932. doi: 10.1063/1.3322586.

[18] Ya. Shpotyuk, C. Boussard-Pledel, V. Nazabal, R. Chahal, J. Ari, B. Pavlyk, J. Cebulski, J.L. Doualan, B. Bureau, Ga-modified $\mathrm{As}_{2} \mathrm{Se}_{3}$-Te glasses for active applications in IR photonics, Optical Materials 46 (2015) 228-232.

[19] Ya. Shpotyuk, B. Bureau, C. Boussard-Pledel, V. Nazabal, R. Golovchak, P. Demchenko, I. Polovynko, Effect of Ga incorporation in the $\mathrm{As}_{30} \mathrm{Se}_{50} \mathrm{Te}_{20}$ glass, J. Non-Cryst. Solids 398-399 (2014) 19. 
[20] I. Kaban, E. Dost, W. Hoyer, Thermodynamic and structural investigations of heat-treated amorphous Ge-Te alloys, J. Alloys and Compounds 379 (2004) 166-170.

[21] A.A. Sherchenkov, S.A. Kozyukhin, E.V. Gorshkova, Transformations in phase-change memory material during thermal cycling, J. Optoelectr. Adv. Materials 11, 1 (2009) 26-33.

[22] Y. Liu, Sh. Yuan, J. Xie, F. Shangguan, J. Ren, G. Chen, A Study on Crystallization Kinetics of Thermoelectric $\mathrm{Bi}_{2} \mathrm{Se}_{3}$ Crystals in $\mathrm{Ge}-\mathrm{Se}-\mathrm{Bi}$ Chalcogenide Glasses by Differential Scanning Calorimeter, J. Am. Ceram. Soc. 96, 7 (2013) 2141-2146.

[23] M.N. Abdel-Rahim, A.Y. Abdel-Latif, A.S. Soltan, Structural study of chalcogenide $\mathrm{Ge}_{20} \mathrm{Se}_{50} \mathrm{Te}_{30}$ glass, Physica B 291 (2000) 41-48.

[24] J.E. Griffiths, G.P. Espinosa, J.C. Phillips, J.P. Remeika, Raman spectra and athermal laser annealing of $\mathrm{Ge}\left(\mathrm{S}_{\mathrm{x}} \mathrm{Se}_{1-\mathrm{x}}\right)_{2}$ glasses, Phys. Rev. B 28 (1983) 4444-4453.

[25] M. M. Abdel-Aziz, Effect of thallium on the crystallization kinetics of the chalcogenide glasses $\mathrm{GeSe}_{2}$ and $\mathrm{GeSe}_{4}$, J. Thermal Analysis and Calorimetry 79 (2005) 709-714.

[26] P.M. Bridenbaugh, G.P. Espinosa, J.E. Griffiths, J.C. Phillips, J.P. Remeika, Microscopic origin of the companion $\mathrm{A}_{1}$ Raman line in glassy Ge(Se,S)2, Phys. Rev. B 20 (1979) 41404144.

[27] T. Ikari, T. Tanaka, K. Ura, K. Moeda, K. Futagami, S. Shigetomi, Raman spectra of P-, Sb- or Bi-doped $\mathrm{Ge}_{\mathrm{x}} \mathrm{Se}_{1-\mathrm{x}}$ glasses, Phys. Rev. B 47 (1993) 4984-4989.

[28] Y. Wang, O. Matsuda, K. Inoue, O. Yamamuro, T. Matsuo, K. Murase, A Raman scattering investigation of the structure of glassy and liquid $\mathrm{Ge}_{\mathrm{x}} \mathrm{Se}_{1-\mathrm{x}}$, J. Non-Cryst. Solids 232-234 (1998) 702-707. 
[29] J. Park, M. Song, S. Yoon, H. Lim, D.S. Jeong, B. Cheong, H. Lee, Structural and optical properties of phase-change amorphous and crystalline $\mathrm{Ge}_{1-\mathrm{x}} \mathrm{Te}_{\mathrm{x}}(0<\mathrm{x}<1)$ thin films, Phys. Status Solidi A 210, 2 (2013) 267-275.

[30] E.F. Steigmeier, G. Harbeke, Soft phonon mode and ferroelectricity in GeTe, Solid State Commun. 8 (1970) 1275-1279.

[31] K.S. Andrikopoulos, S.N. Yannopoulos, A.V. Kolobov, P. Fons, J. Tominaga, Raman scattering study of $\mathrm{GeTe}$ and $\mathrm{Ge}_{2} \mathrm{Sb}_{2} \mathrm{Te}_{5}$ phase-change materials, J. Phys. Chem. Solids 68 (2007) 1074-1078.

[32] K.S. Andrikopoulos, S.N. Yannopoulos, G.A. Voyiatzis, A.V. Kolobov, M. Ribes, J. Tominaga, Raman scattering study of the a-GeTe structure and possible mechanism for the amorphous to crystal transition, J. Phys.: Condens. Matter 18 (2006) 965-979.

[33] P. Nemec, V. Nazabal, A. Moreac, J. Gutwirth, L. Benes, M. Frumar, Amorphous and crystallized Ge-Sb-Te thin films deposited by pulsed laser: Local structure using Raman scattering spectroscopy, Materials Chemistry and Physics 136 (2012) 935-941.

[34] M. Von der Emde, D.R.T. Zahn, T. Ng, N. Maung, G.H. Fan, I.B. Poole, J.O. Williams, A.C. Wright, MOCVD growth of $\mathrm{Ga}_{2} \mathrm{Se}_{3}$ on $\mathrm{GaAs}(100)$ and $\mathrm{GaP}(100)$ : a Raman study, Applied Surface Science 104/105 (1996) 575-579.

[35] A. W. Mao, B. G. Aitken, R. E. Youngman, D. C. Kaseman, S. Sen, Structure of Glasses in the Pseudobinary System $\mathrm{Ga}_{2} \mathrm{Se}_{3}-\mathrm{GeSe}_{2}$ : Violation of Chemical Order and $8-\mathrm{N}$ Coordination Rule, J. Phys. Chem. B 117 (2013) 16594-16601.

[36] I. Voleska, J. Akola, P. Jovari, J. Gutwirth, T. Wagner, Th. Vasileiadis, S. N. Yannopoulos, R. O. Jones, Structure, electronic, and vibrational properties of glassy Ga11Ge11Te78: Experimentally constrained density functional study, Phys. Rev. B 86 (2012) 094108. 
[37] E. Mytilineou, B.S. Chao, D. Papadimitriou, Raman scattering in sputtered amorphous $\mathrm{Ge}_{25} \mathrm{Se}_{75-\mathrm{x}} \mathrm{Bi}_{\mathrm{x}}$ films, J. Non-Cryst. Solids 195 (1996) 279-285.

[38] J. Zhang, G. Huang, Phonon dynamics in $\left(\mathrm{Bi}_{2} \mathrm{Se}_{3}\right)_{\mathrm{m}}\left(\mathrm{Bi}_{2}\right)_{\mathrm{n}}$ infinitely adaptive series, Solid State Communications 197 (2014) 34-39.

[39] J.O. Jenkins, J.A. Rayne, R. W. Ure, Elastic Moduli and Phonon Properties of $\mathrm{Bi}_{2} \mathrm{Te}_{3}$, Phys. Rev. B 5 (1972) 3171.

[40] L.M. Goncalves, C. Couto, P. Alpuim, A. G. Rolo , F. Völklein, J. H. Correia, Optimization of thermoelectric properties on $\mathrm{Bi}_{2} \mathrm{Te}_{3}$ thin films deposited by thermal coevaporation, Thin Solid Films 518 (2010) 2816.

[41] W. Kullmann, J. Geurts, W. Richter, N. Lehner, H. Rauh, U. Steigenberger, G. Eichhorn, R. Geick, Effect of Hydrostatic and Uniaxial Pressure on Structural Properties and Raman Active Lattice Vibrations in $\mathrm{Bi}_{2} \mathrm{Te}_{3}$, Phys. Status Sol. (b) 125 (1984) 131.

[42] ICDD (2015). PDF-4+ 2015 (Database), edited by Dr. Soorya Kabekkodu, International Centre for Diffraction Data, Newtown Square, PA, USA.

[43] T. Ozawa, Nonisothermal kinetics of crystal growth from pre-existing nuclei, Bull. Chem. Soc. Jpn. 57 (1984) 639-643.

[44] T. Ozawa, A new method of analyzing thermogravinetric data, Bull. Chem. Soc. Japan 38 (1965) 1881-1886. J. H. Flynn, L. A. Wall, A quick, direct method for the determination of activation energy from thermogravimetric data, Polym. Lett. 4 (1966) 323-328.

[45] J. Malek, Kinetic analysis of crystallization processes in amorphous materials, Thermochimica Acta 355 (2000) 239-253.

[46] J. Sestak, Thermophysical Properties of Solids. Their Measurements and Theoretical Analysis, Elsevier, Amsterdam, 1984. 
[47] D.W. Henderson, Experimental analysis of non-isothermal transformations involving nucleation and growth, J. Therm. Anal. 15 (1979) 325-331.

[48] D.W.Henderson, Thermal analysis of non-isothermal crystallization kinetics in glass forming liquids, J. Non-Cryst. Solids 30 (1979) 301-315.

[49] K. Matusita, T. Komatsu, R. Yokota, Kinetics of non-isothermal crystallization process and activation energy for crystal growth in amorphous materials, J. Mater. Sci. 19 (1984) 291294.

[50] J. Malek, The applicability of Johnson-Mehl-Avrami model in the thermal analysis of the crystallization kinetics of glasses, Thermochimica Acta 267 (1995) 61-73.

[51] R. Svoboda, V. Karabyn, J. Málek, M. Frumar, L. Beneš, M. Vlček, Amorphous-tocrystalline transition in $\mathrm{Ge}_{8} \mathrm{Sb}_{(2-\mathrm{x})} \mathrm{Bi}_{\mathrm{x}} \mathrm{Te}_{11}$ phase-change materials for data recording, $\mathrm{J}$. Alloys and Compounds (2015) in press. 


\section{Figure captions}

Fig. 1. (a) $\mathrm{XRD}$ patterns of $\mathrm{Bi}_{1} \mathrm{Ga}_{5} \mathrm{Ge}_{18.8} \mathrm{Se}_{37.6} \mathrm{Te}_{37.6}$ (R1), $\mathrm{Bi}_{5} \mathrm{Ga}_{5} \mathrm{Ge}_{18} \mathrm{Se}_{36} \mathrm{Te}_{36}$ (R2), $\mathrm{Bi}_{10} \mathrm{Ga}_{5} \mathrm{Ge}_{17} \mathrm{Se}_{34} \mathrm{Te}_{34}$ (R3) samples and $\mathrm{Bi}_{2} \mathrm{Se}_{3}, \mathrm{Bi}_{2} \mathrm{Te}_{3}$ crystals; (b) SEM picture of the fresh crack of as-prepared R3 sample.

Fig. 2. DSC curves recorded at different heating rates for $\mathrm{Bi}_{1} \mathrm{Ga}_{5} \mathrm{Ge}_{18.8} \mathrm{Se}_{37.6} \mathrm{Te}_{37.6}$ (R1), $\mathrm{Bi}_{5} \mathrm{Ga}_{5} \mathrm{Ge}_{18} \mathrm{Se}_{36} \mathrm{Te}_{36}(\mathrm{R} 2)$ and $\mathrm{Bi}_{10} \mathrm{Ga}_{5} \mathrm{Ge}_{17} \mathrm{Se}_{34} \mathrm{Te}_{34}(\mathrm{R} 3)$ samples.

Fig. 3. Example of Gaussian (left column) and Fraser-Suzuki (right column) fittings for DSC curves of $\mathrm{Bi}_{1} \mathrm{Ga}_{5} \mathrm{Ge}_{18.8} \mathrm{Se}_{37.6} \mathrm{Te}_{37.6}(\mathrm{R} 1), \mathrm{Bi}_{5} \mathrm{Ga}_{5} \mathrm{Ge}_{18} \mathrm{Se}_{36} \mathrm{Te}_{36}(\mathrm{R} 2)$ and $\mathrm{Bi}_{10} \mathrm{Ga}_{5} \mathrm{Ge}_{17} \mathrm{Se}_{34} \mathrm{Te}_{34}(\mathrm{R} 3)$ samples recorded at $5 \mathrm{~K} / \mathrm{min}$ heating rate.

Fig. 4. Raman spectra of the investigated R1 (a), R2 (b) and R3 (c) samples before (glassy state) and after (crystallized) heat treatment at different temperatures. Fitting is performed with minimum number of Gaussians as guide for the eyes.

Fig. 5. XRD spectra of the investigated $R 1, R 2$ and $R 3$ samples after heat treatment (crystallization) at $250{ }^{\circ} \mathrm{C}, 300{ }^{\circ} \mathrm{C}$ and $400{ }^{\circ} \mathrm{C}$ as indicated.

Fig. 6. Ozawa's $[43,44]$ plots for $\mathrm{Bi}_{1} \mathrm{Ga}_{5} \mathrm{Ge}_{18.8} \mathrm{Se}_{37.6} \mathrm{Te}_{37.6}(\mathrm{R} 1)$ sample to determine activation energies $\left(E_{a}\right)$ for the crystallization processes as per Gaussian (solid symbols) and Fraser-Suzuki (open symbols) fits.

Fig. 7. Ozawa's $[43,44]$ plots for $\mathrm{Bi}_{5} \mathrm{Ga}_{5} \mathrm{Ge}_{18} \mathrm{Se}_{36} \mathrm{Te}_{36}$ (R2) sample to determine activation energies $\left(E_{a}\right)$ for the crystallization processes as per Gaussian (solid symbols) and Fraser-Suzuki (open symbols) fits. 
Fig. 8. Ozawa's $[43,44]$ plots for $\mathrm{Bi}_{10} \mathrm{Ga}_{5} \mathrm{Ge}_{17} \mathrm{Se}_{34} \mathrm{Te}_{34}$ (R3) sample to determine activation energies $\left(E_{a}\right)$ for the crystallization processes as per Gaussian (solid symbols) and Fraser-Suzuki (open symbols) fits.

Fig. 9. $z(\alpha)$ functions for "high-temperature" crystallization region of R1-R3 samples to check the applicability of JMA equation. Full symbols correspond to the peak V in Fraser-Suzuki fit, open symbols - experimental datapoints.

Fig. 10. Plots for JMA exponent determination for the peak $V$ of R1-R3 samples using experimental datapoints (open symbols) and Fraser-Suzuki fit (solid symbols). 
TABLE

Table 1. Dietzel criterion of glass stability [12] and index of crystallization rapidity ICR [51], calculated for the investigated ChG using DSC curves recorded at $10 \mathrm{~K} /$ min heating rate.

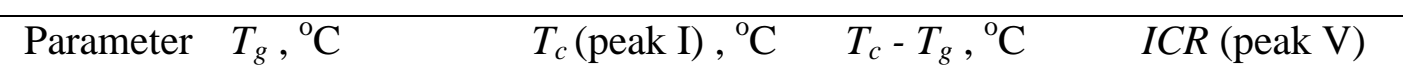

Sample $\quad( \pm 1) \quad( \pm 1)$

\begin{tabular}{lllll}
\hline $\mathrm{Bi}_{1} \mathrm{Ga}_{5} \mathrm{Ge}_{18.8} \mathrm{Se}_{37.6} \mathrm{Te}_{37.6}$ & 174 & 245 & 71 & 0.124 \\
\hline $\mathrm{Bi}_{5} \mathrm{Ga}_{5} \mathrm{Ge}_{18} \mathrm{Se}_{36} \mathrm{Te}_{36}$ & 186 & 230 & 44 & 0.120 \\
\hline $\mathrm{Bi}_{10} \mathrm{Ga}_{5} \mathrm{Ge}_{17} \mathrm{Se}_{34} \mathrm{Te}_{34}$ & 215 & partially crystallized & -0.011 \\
\hline
\end{tabular}


FIGURES

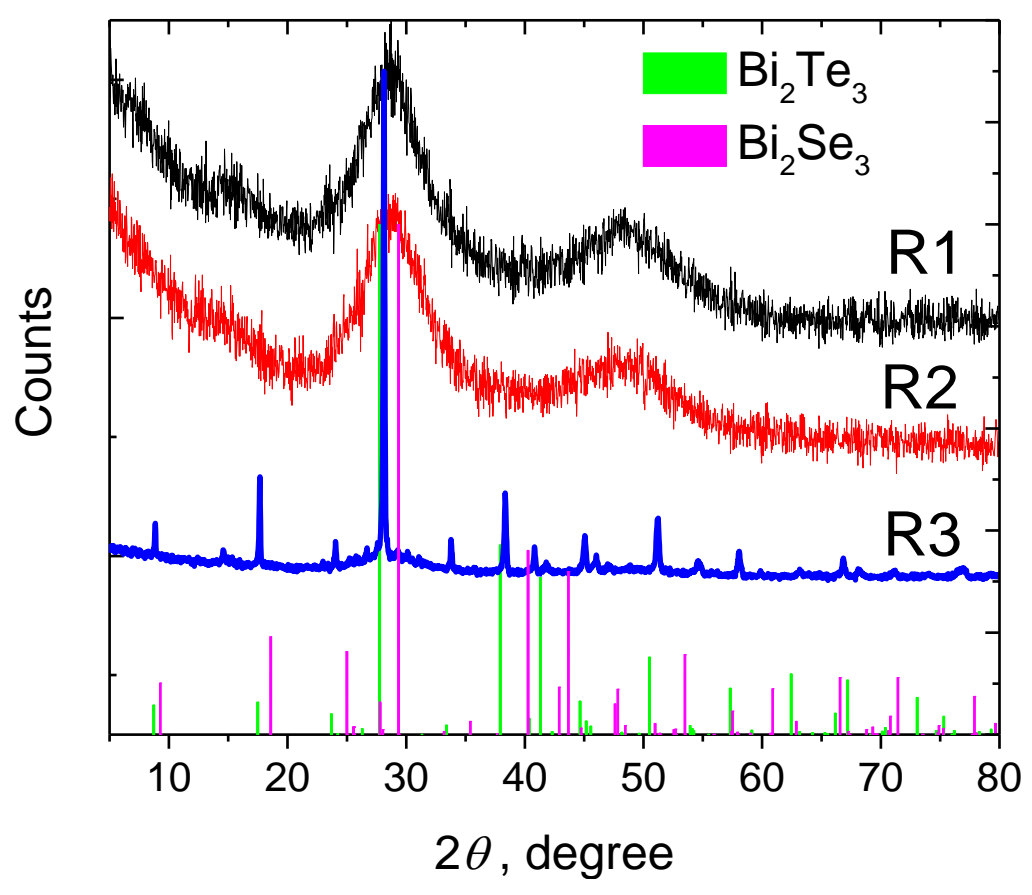

(b)

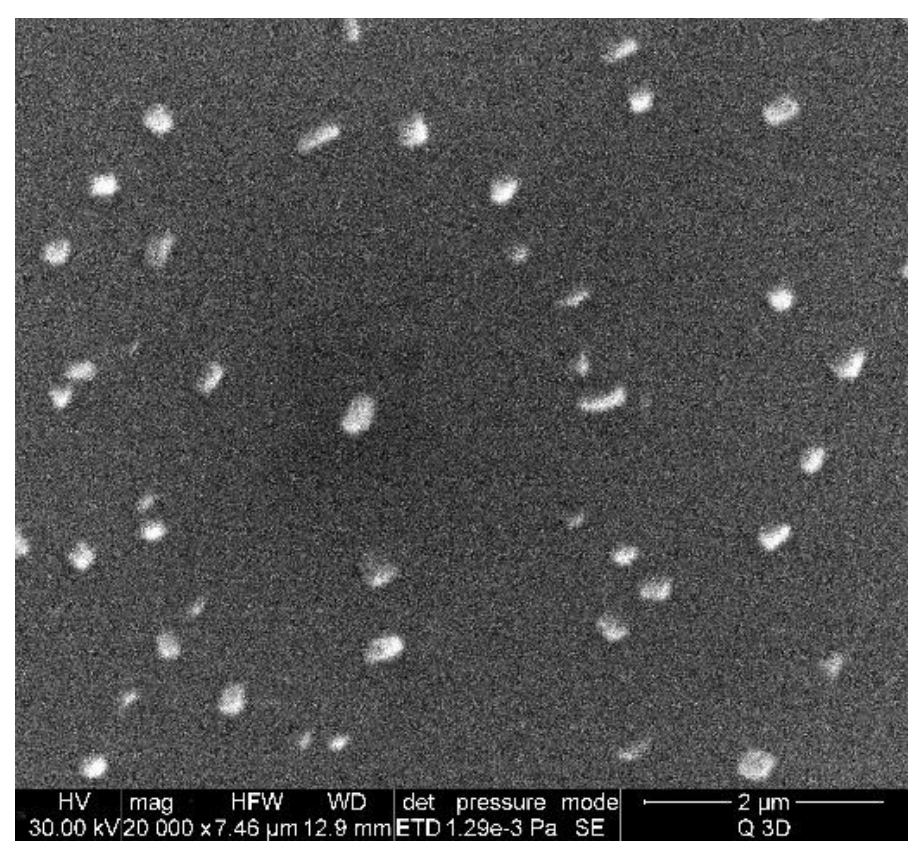

Fig. 1. 


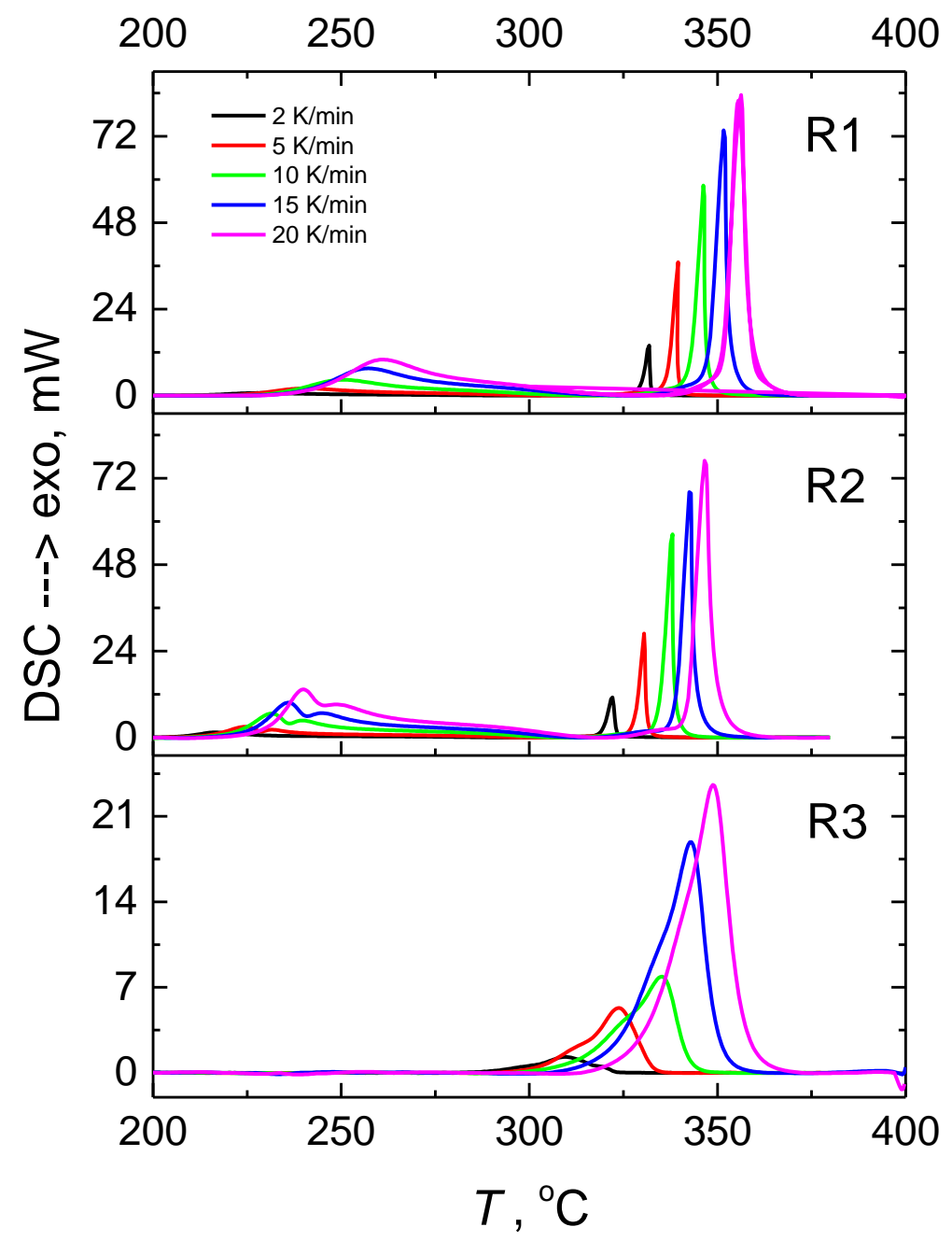

Fig. 2. 

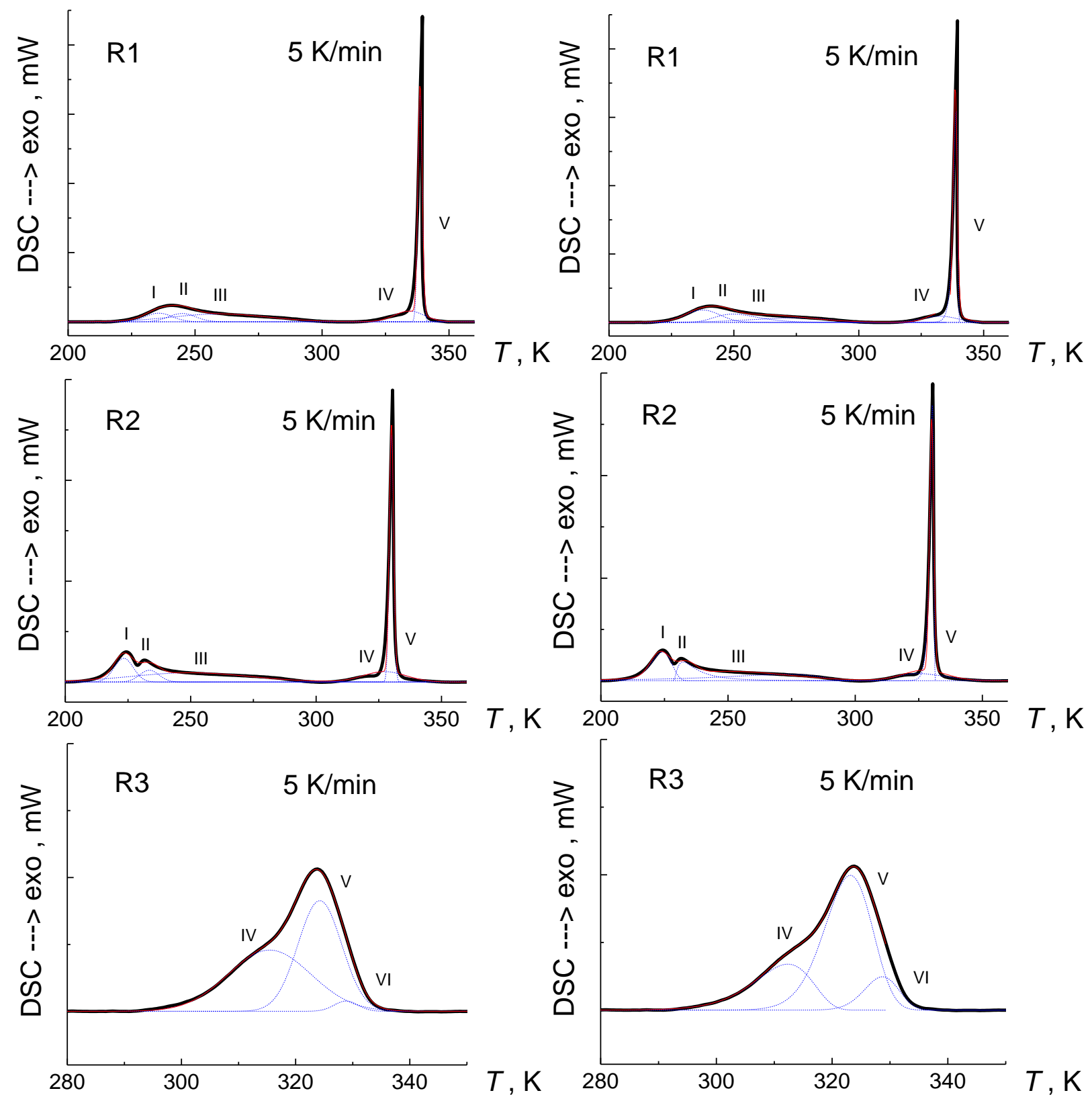

Fig. 3. 
(a)

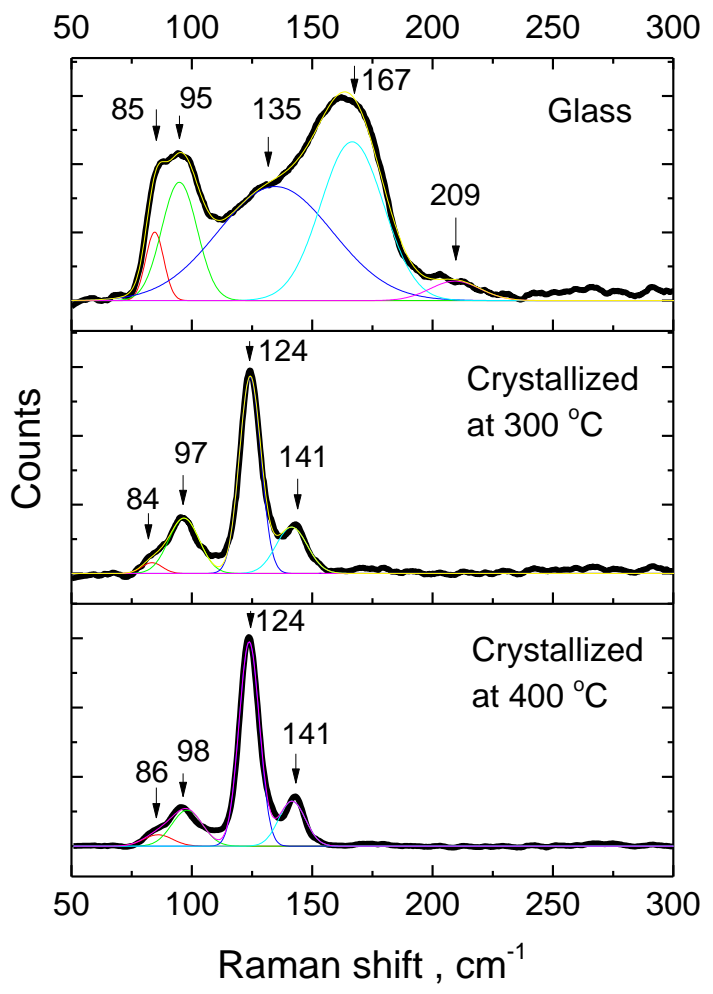

(b)

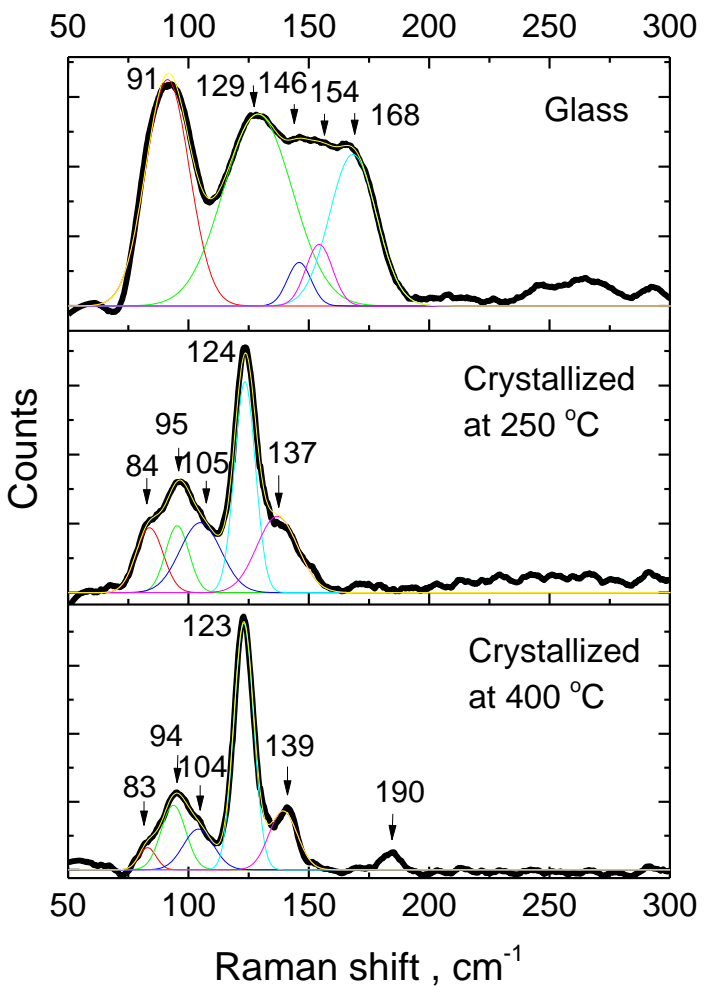

(c)

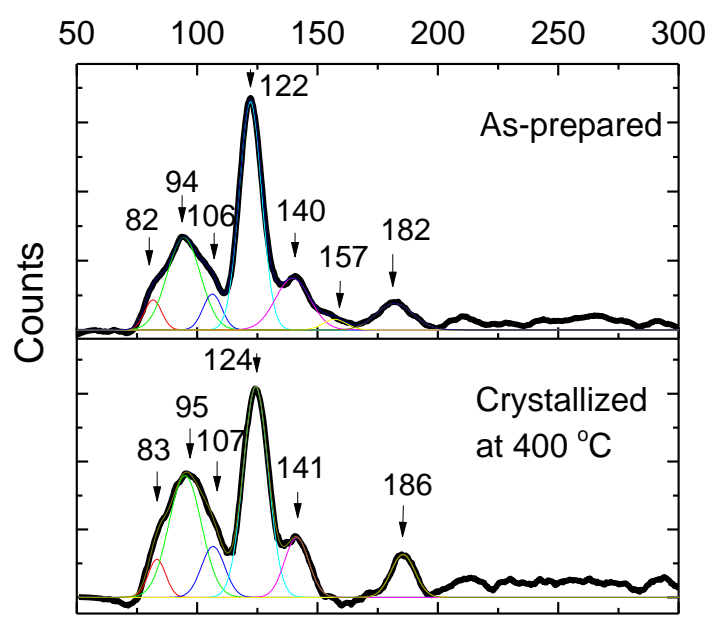

Fig. 4. 


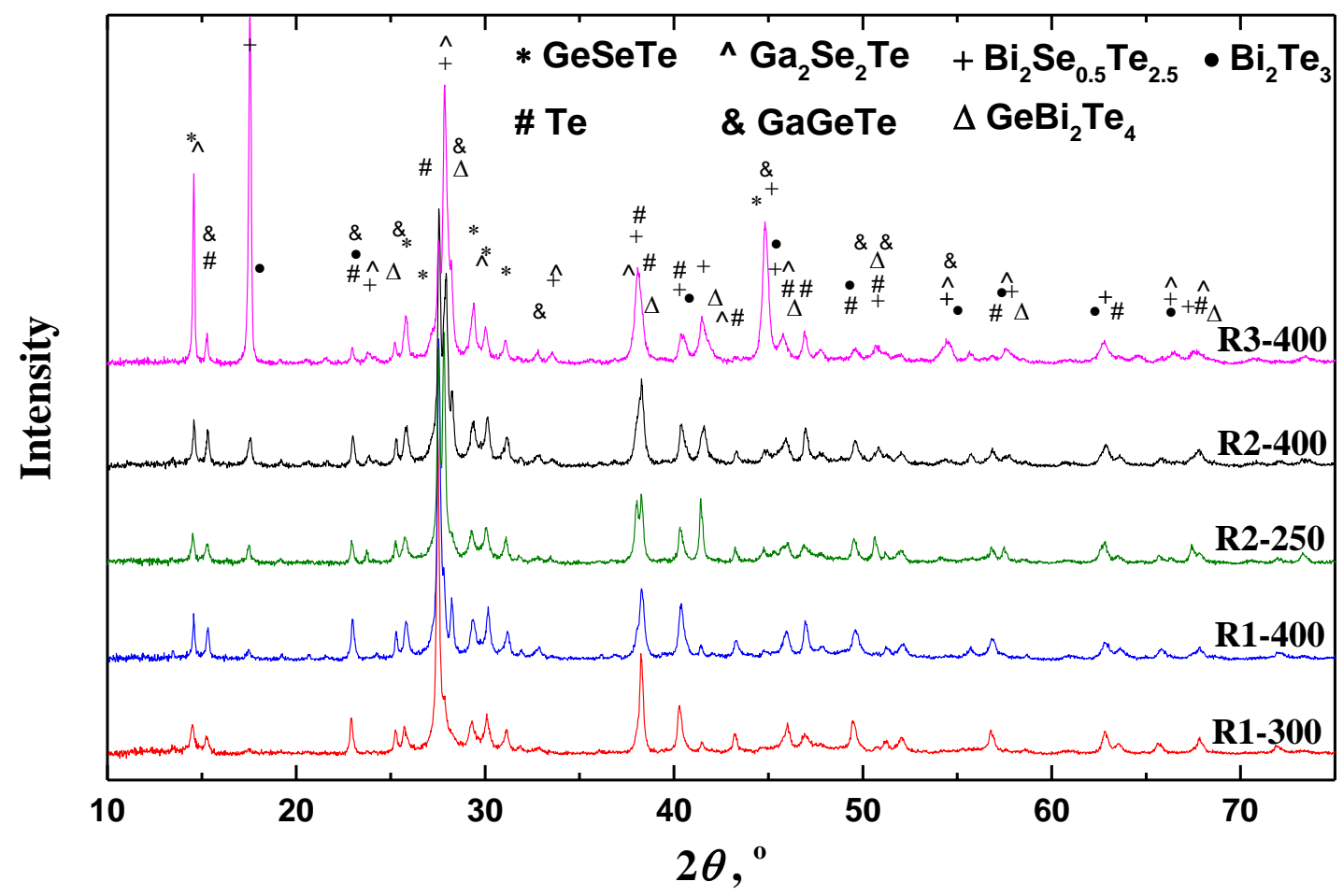

Fig. 5. 

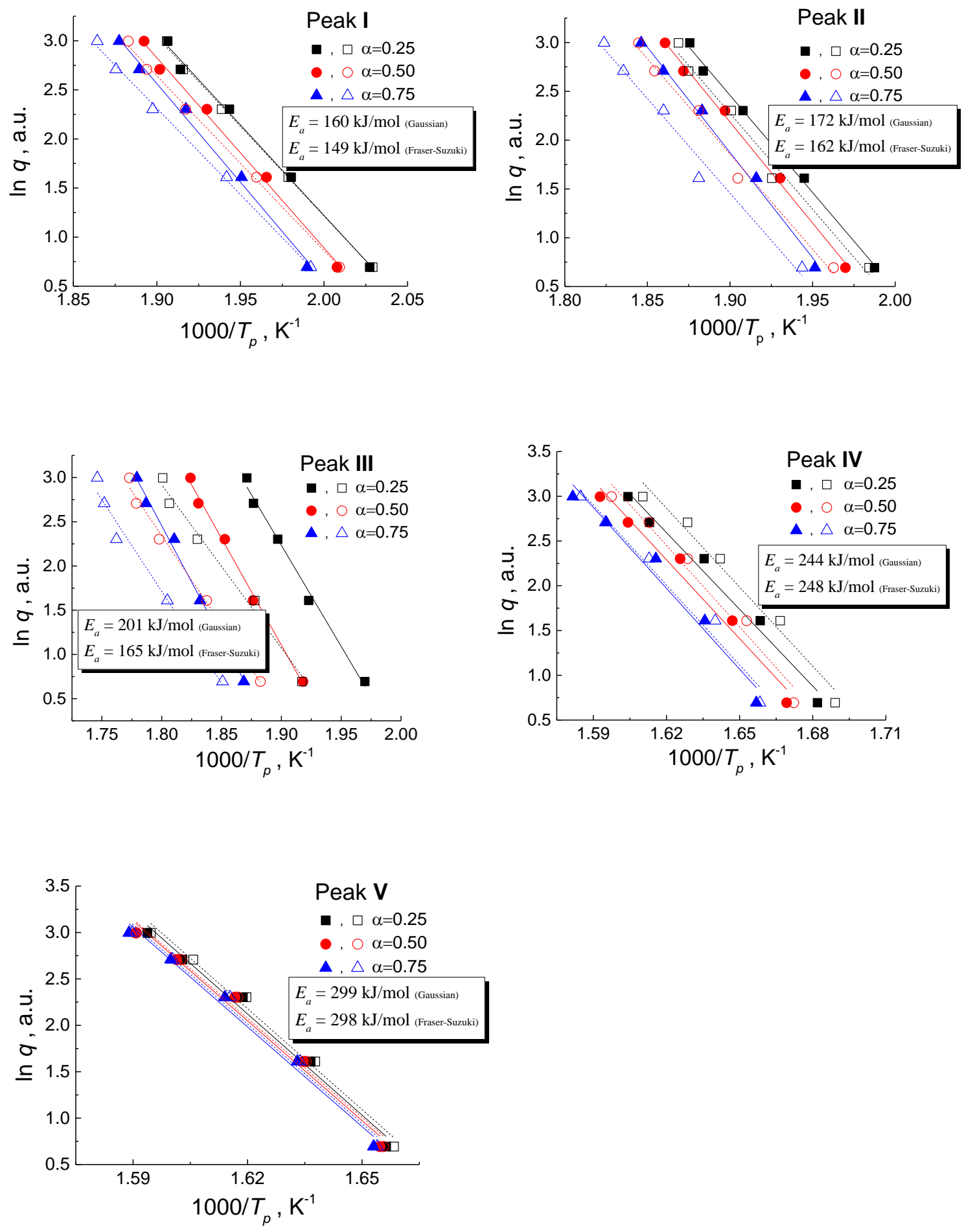

Fig. 6. 

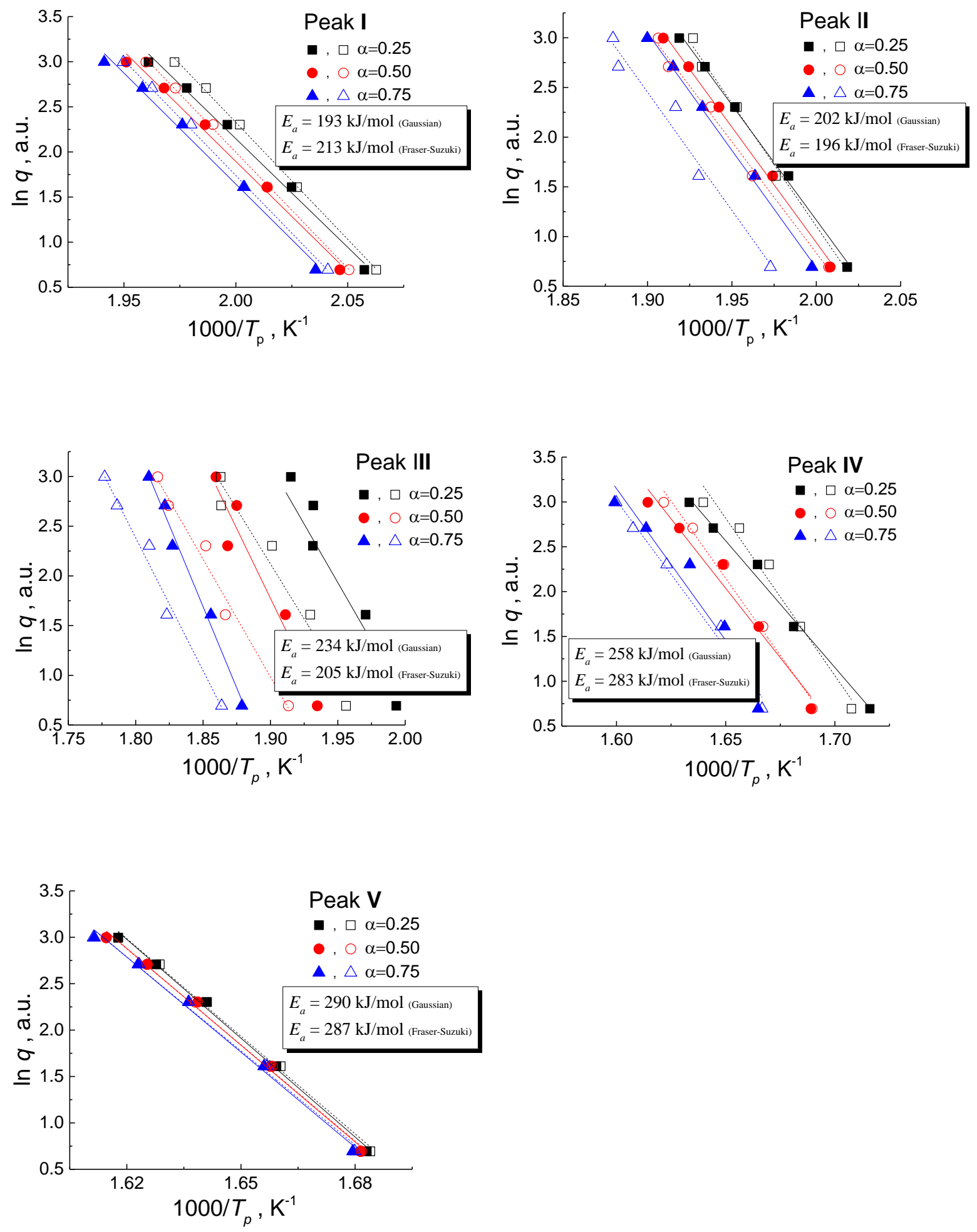

Fig. 7. 

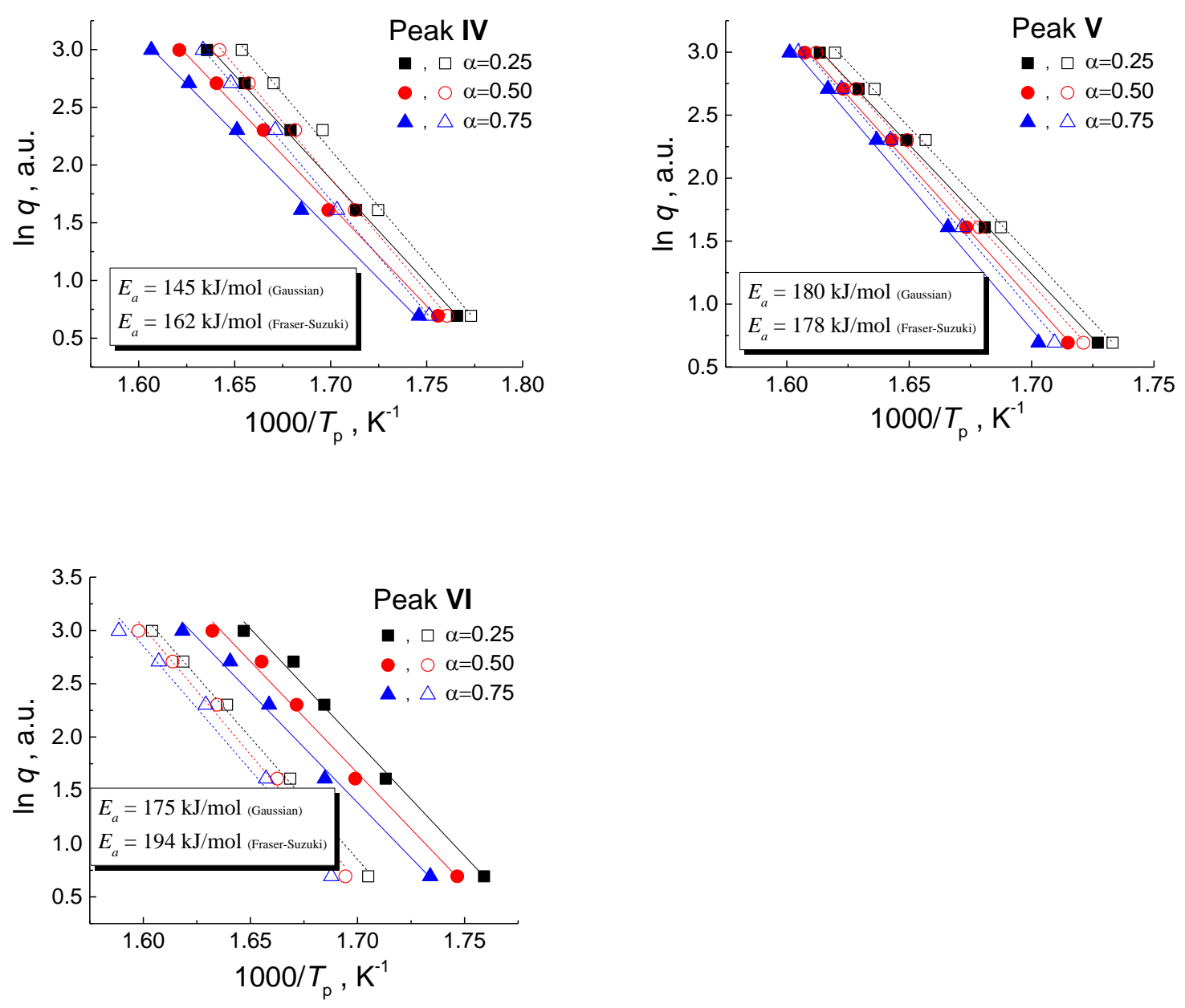

Fig. 8. 

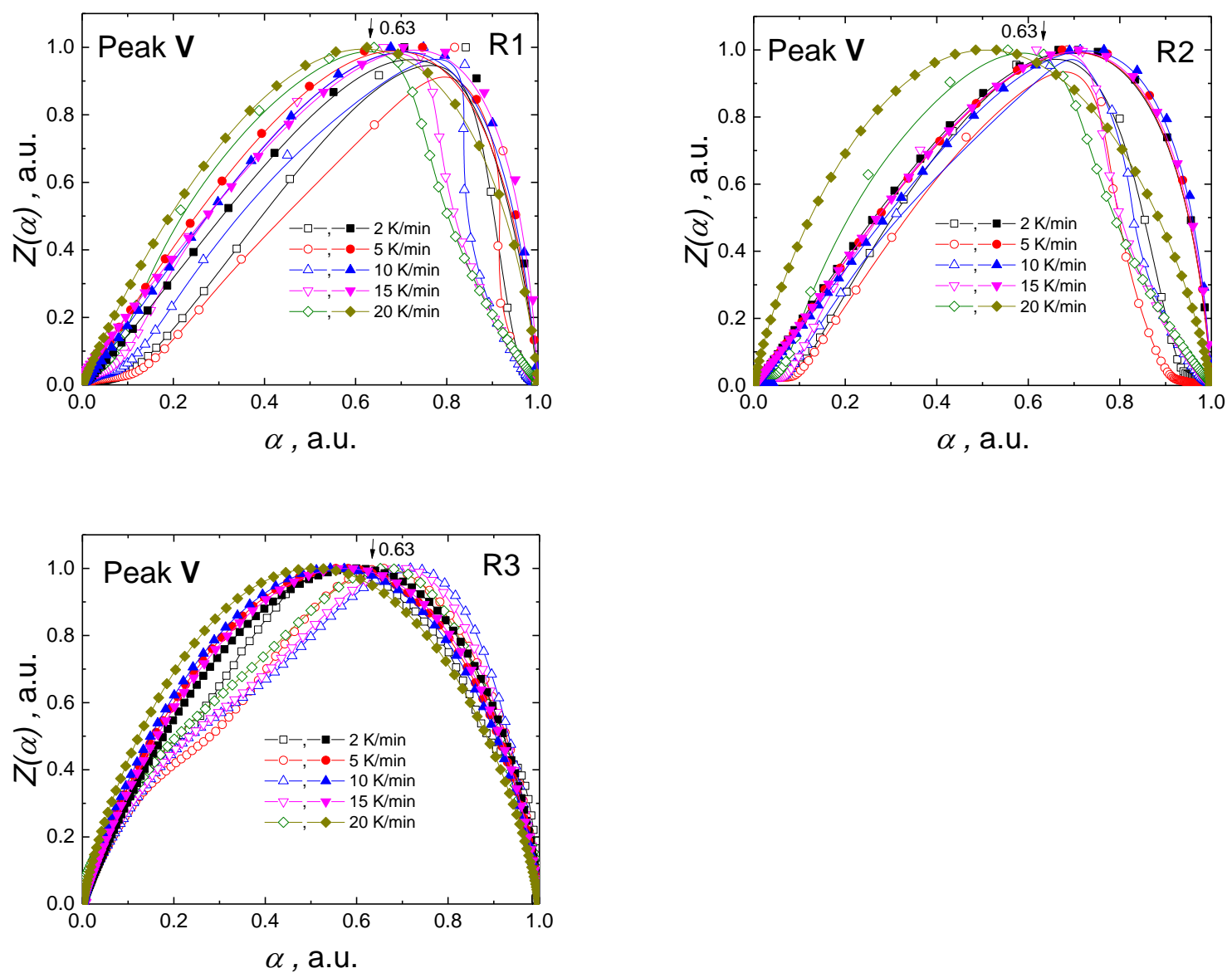

Fig. 9. 

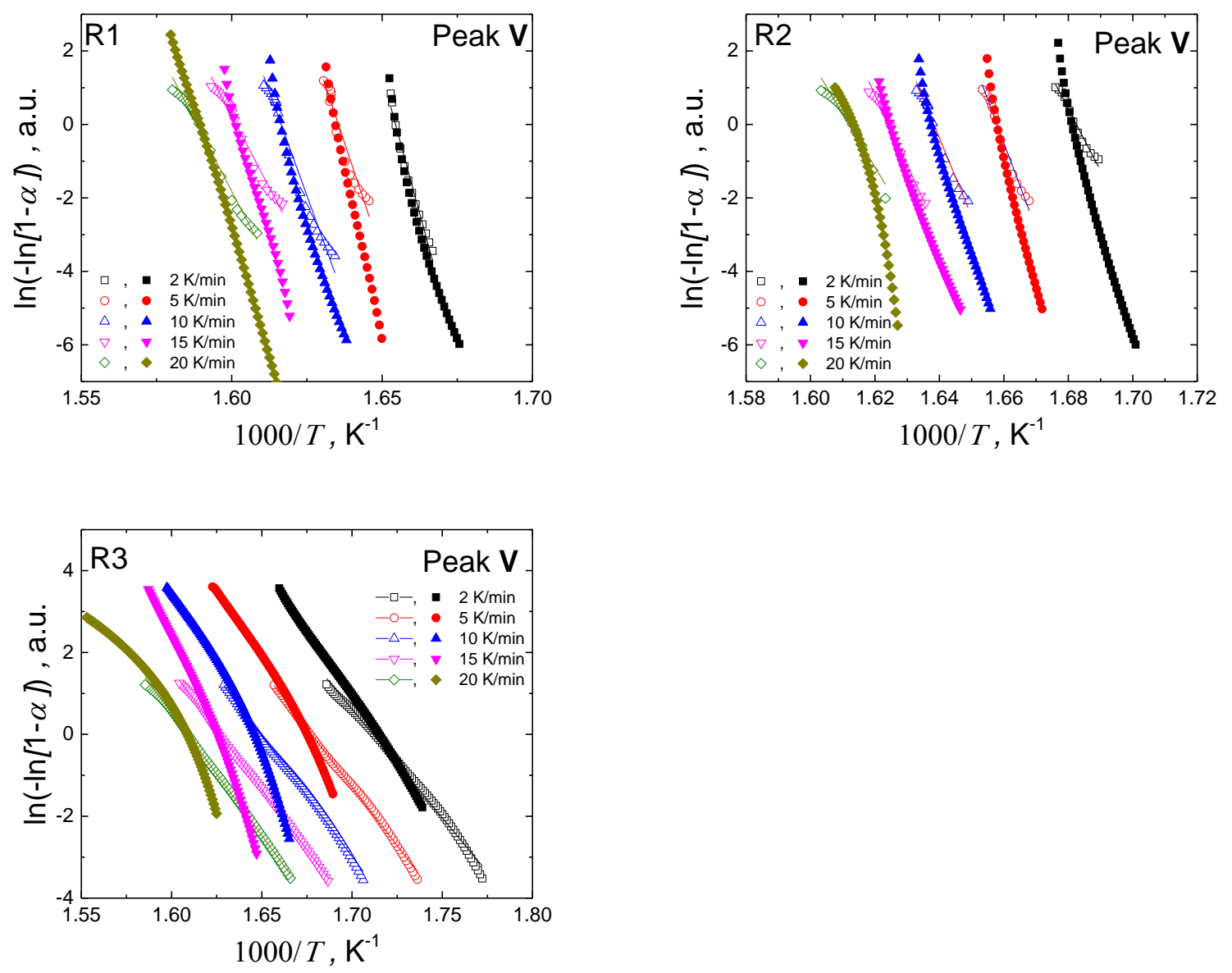

Fig. 10. 